\title{
Developing new neurophysiological signatures of general anesthesia induced loss of consciousness
}

\author{
Aylin Cimenser*1,2,3, Eric T Pierce ${ }^{1,2}$, Andres F Salazar Gomez ${ }^{1,3}$, \\ John Walsh1,2, Priscilla G Harrell1,2, Casie L Tavares' ${ }^{1}$, Kathleen Habeeb ${ }^{1}$, \\ Patrick Purdon ${ }^{1,2,3}$ and Emery N Brown ${ }^{1,2,3}$
}

\author{
Address: ${ }^{1}$ Department of Anesthesia and Critical Care, Massachusetts General Hospital, Boston, MA, 02114, USA, ${ }^{2}$ Harvard Medical School, \\ Boston, MA, 02115, USA and ' 3 Department of Brain and Cognitive Science, Massachusetts Institute of Technology, Cambridge, MA, 02139, USA \\ Email: Aylin Cimenser* - aylin@nmr.mgh.harvard.edu \\ * Corresponding author
}

from Eighteenth Annual Computational Neuroscience Meeting: CNS*2009

Berlin, Germany. 18-23 July 2009

Published: 13 July 2009

BMC Neuroscience 2009, I0(Suppl I):P79 doi:I0.I |86/I47|-2202-I0-SI-P79

This abstract is available from: http://www.biomedcentral.com/I47I-2202/I0/SI/P79

(c) 2009 Cimenser et al; licensee BioMed Central Ltd.

General anesthesia is currently defined in the context of its use in clinical care [1]. The mechanism by which anesthetic drugs induce general anesthesia is not well understood. There is therefore a need to incorporate neurophysiological characterizations into the definition and understanding of anesthesia. Distinct patterns in the electroencephalogram have been associated with anesthesia-induced loss of consciousness [2-4] and are used as part of protocols to monitor integrity of brain function [5]. However, the transition to unconsciousness during a gradual induction of general anesthesia has not been studied systematically.

Here we report results of multivariate frequency-domain characterizations of propofol induced changes in the scalp EEG of human subjects performing a behavioral task. In this task subjects were asked to respond to auditory stimuli while loss of consciousness was induced through gradual increase in propofol dosage. Subsequently, the dose was decreased and the subjects recovered consciousness. EEG was recorded from 64 channels sampled at $5000 \mathrm{~Hz}$. Behavioral data was gathered throughout the recording period and subjects were required to keep their eyes closed.
We characterize the temporal dynamics of the EEG through an eigenvalue decomposition of the time and frequency dependent cross spectral matrix. We find that the state of anesthesia induced unconsciousness as assessed from behavioral data is strongly correlated with the persistence of a single dominant mode in the high alpha low beta range (see Figure 1) concentrated in the frontal channels. As the propofol dose was decreased the contribution of this mode decreased as well. Our findings suggest that general anesthesia induced unconsciousness may be accompanied by a single dominant mode at selected frequencies between the frontal channels.

\section{Acknowledgements}

Supported by the NIH DPI OD003646 (ENB), K25-NS05758 (PP) and GCRC I ULI RR025758-0I.

\section{References}

I. Stoelting RK, Miller RD: Basics of Anesthesia 5th edition. Philadelphia, PA: Elsevier; 2007.

2. Rampil IJ, Matteo RS: Changes in EEG spectral edge frequency correlate with the hemodynamic response to laryngoscopy and intubation. Anesthesiology 1987, 67:139-142.

3. Rampil IJ: A primer for EEG signal processing in anesthesia. Anesthesiology 1998, 89:980-1002.

4. John ER, Prichep LS: The anesthetic cascade: a theory of how anesthesia suppresses consciousness. Anesthesiology 2005, 102:447-47|. 


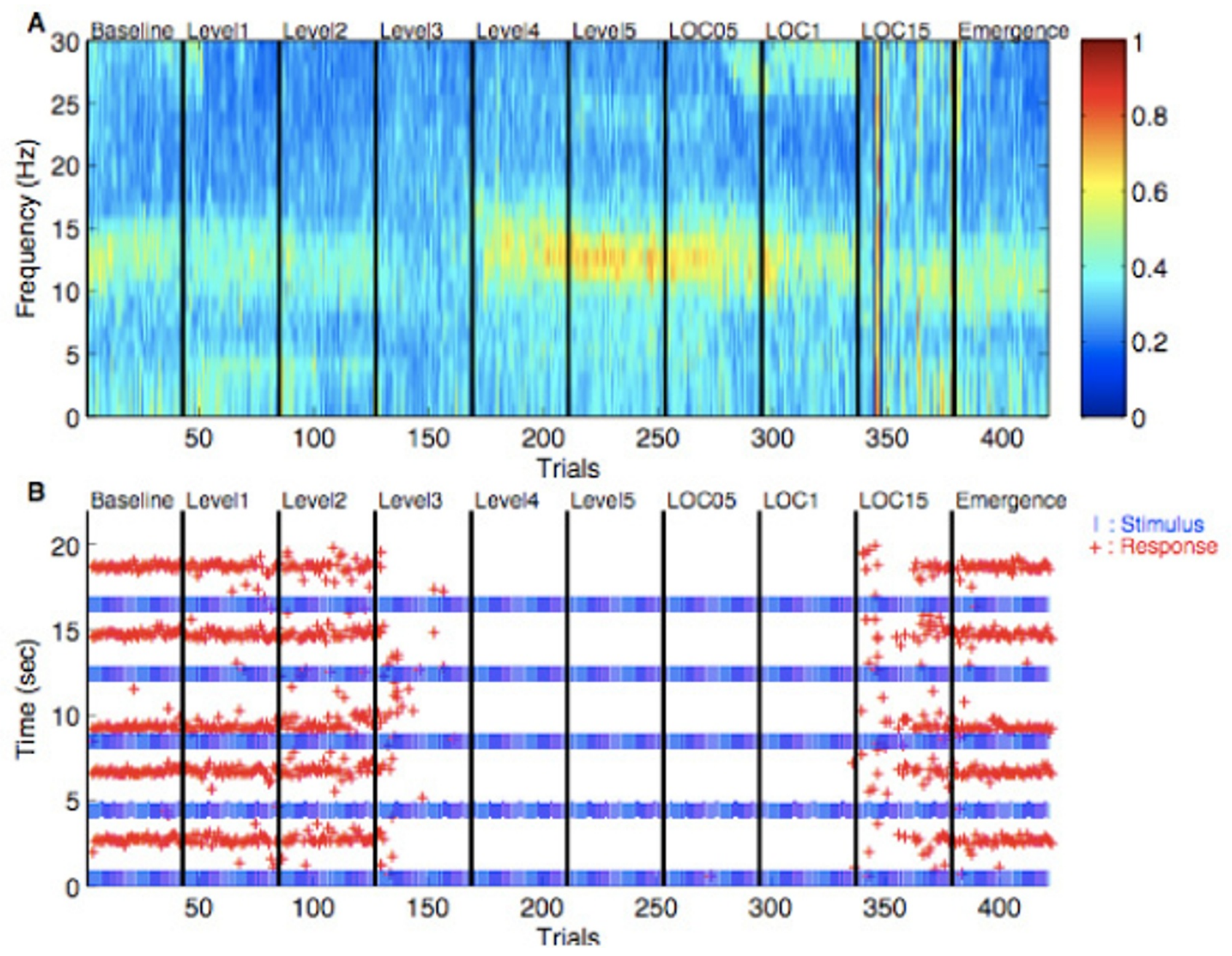

Figure I

(A) Fraction of total energy contained in the leading eigenmode. (B) Behavioral data.

5. Kearse Jr, et al: Somatosensory evoked potentials sensitivity relative to electroencephalography for cerebral ischemia during carotid endarterectomy. Stroke 1992, 23:498-505.

Publish with Biomed Central and every scientist can read your work free of charge

"BioMed Central will be the most significant development for disseminating the results of biomedical research in our lifetime." Sir Paul Nurse, Cancer Research UK

Your research papers will be:

- available free of charge to the entire biomedical community

- peer reviewed and published immediately upon acceptance

- cited in PubMed and archived on PubMed Central

- yours - you keep the copyright
BioMedcentral 